\title{
A Review of Patients in a Psychosexual Clinic-How Strong is the Relation- ship between Psychosexual Problems and a History of Sexual Abuse or Assault?
}

Lucy Frances Love*

Clinical Director, G4s Forensic and Medical Service, UK

\begin{abstract}
The notes of 100 patients who attended a psychosexual clinic in the UK between 2011 and 2013 were analysed. The notes were searched for a disclosure of sexual abuse or assault and the frequency of disclosure was calculated. Patients with an obvious physical cause for their psychosexual problem, such as diabetes were excluded. Patients presented with a wide range of psychosexual problems and the relationship between sexual abuse/assault and the specific type of psychosexual problem was also explored. The results of this study, although small, could possibly support more financial investment into initial counselling and domestic/sexual violence services to prevent the development of serious long term psychosexual sequelae, as these can be very costly to treat, both in terms of finance and clinic time.
\end{abstract}

\section{Aim}

To explore the relationship between a history of sexual abuse/ assault and the subsequent development of psychosexual problems.

\section{Method}

The setting for this study was a weekly NHS psychosexual clinic, within a family planning service in Medway, Kent, UK. Demographic data for the Medway urban conurbation suggest a 93\% British White population. The clinic was run by a single doctor and the notes made by the doctor during the counselling sessions of 100 patients seen from 2011 to 2013 were analysed and a search performed for a disclosure of sexual abuse or assault. Patients with an obvious physical cause for their psychosexual problem such as men with diabetes causing erectile dysfunction or women with the skin disorder, lichen sclerosus causing dyspareunia (painful sex), were excluded. The frequency with which sexual abuse or assault had been disclosed was calculated as a percentage of the patient notes reviewed. There were 17 males and 83 females in the study, which falls within the usual range of male to female patients seen within the Medway psychosexual clinic. The age range was 2373 years for males and 16-59 for females. Mean age for women was 5 years and for men 36 years. A wide variety of psychosexual problems were reported and an attempt was made to investigate the relationship between a disclosure of abuse and the specific type of psychosexual problem reported.

\section{Results}

The notes of 100 patients ( 83 female and 17 male), without an obvious physical cause for their psychosexual problem and who attended the psychosexual clinic between 2011 and 2013 were reviewed and 59 (59\%) were found to have voluntarily reported either a past history of child sexual abuse or a sexual assault. Of the 59 patients reporting sexual abuse or assault, 11 were male and 48 were female. I then looked at the particular psychosexual problem that patients had presented with. The psychosexual problems included:

Females: Vaginismus (vaginal muscle spasm), dyspareunia (painful sex), loss of libido, phobia of STIs, pelvic pain, anorgasmia, body dysmorphia (labia of vulva perceived to be abnormal).

Males: Erectile dysfunction, premature ejaculation, sexual addiction, retarded or non-ejaculation, loss of sensation, loss of libido.
Of these 59 patients with a psychosexual problem and a history of child sexual abuse (CSA) or sexual assault, 48 were female and 26 of these 48 patients (over 50\%) were complaining of vaginismus or dyspareunia and 21 had loss of libido.

11 of the 59 were male and 5 of these 11 went on to develop a sexual addiction.

\section{Discussion}

The percentage of patients reporting sexual abuse or a sexual assault was almost $60 \%$ (59\%) and this could well be an under-estimate as patients were not always asked a direct question as to whether they had been abused or assaulted and they might have been reluctant to disclose the information, if they had never previously reported it to their family or the police.

This was a small study and demographic variables such as race, ethnicity, age, sex and social status were not controlled for. The Medway population is $93 \%$ British White and $1.5 \%$ Asian and Medway is an area of high deprivation, with high rates of unemployment and high teenage pregnancy rates.

There was a younger age range of females (16-59years) attending the psychosexual clinic than males (23-73years) and there were 20 women in the study under the age of 25 years in comparison to only 1 male under 25 years. 14 of the women with a history of sexual abuse were under the age of 25 years, whereas none of the males with a history of sexual abuse were under 25 years. These findings could simply reflect the fact that women may be quicker and more willing to present with a health problem than men, as has been reported to occur in general

*Corresponding author: Lucy Frances Love,, Clinical Director, G4s Forensic and Medical Service, UK, Tel: (44) 07966234991; E-mail: Ifclove@yahoo.com

Received May 05, 2014; Accepted June 26, 2014; Published June 30, 2014

Citation: Love LF (2014) A Review of Patients in a Psychosexual Clinic-How Strong is the Relationship between Psychosexual Problems and a History of Sexual Abuse or Assault? J Forensic Res 5: 236 doi:10.4172/2157-7145.1000236

Copyright: @ 2014 Love LF. This is an open-access article distributed under the terms of the Creative Commons Attribution License, which permits unrestricted use, distribution, and reproduction in any medium, provided the original author and source are credited. 
practise in the UK [1] or possibly women were exhibiting psychosexual problems from the very first time they had sex following the abuse, whereas men were only revealing their psychosexual problems later in life when sexual arousal was less automatic and erectile dysfunction more common, so psychological factors might then impact more on their sexual functioning. It could also be that women had less choice of when they became sexually active whereas men, who had been sexually abused, were choosing to wait until they were older to have their first sexual experience following the abuse.

A significant finding which emerged from this study, although not statistically significant was that a common presenting psychosexual problem for women was painful sex (dyspareunia) or an inability to allow penetrative sex due to muscle spasm (vaginismus), usually because they had extreme anxiety or a fear of sex, whereas for men, the consequence of the sexual abuse appeared from this small study to be the opposite and caused them to become overtly sexualised and develop a sexual addiction.

\section{Conclusion}

The results of this small study do show that a high percentage of patients attending a psychosexual clinic (almost 60\%) had a history of sexual abuse or assault. For women the most common psychosexual problems they presented with were dyspareunia (painful sex), vaginismus (vaginal muscle spasm) or loss of libido whereas for men it was a sexual addiction. Many of these patients had never previously told anyone about the abuse, so any method to encourage victims to disclose and seek help, prior to developing a psychosexual problem, should be sought [2-5]. Such measures could include more financial support for the development of sexual assault referral centres where victims can self-refer for advice and support, increasing the number of ISVAs (independent sexual violence advisors) and IDVAs (Independent domestic violence advisors) to support victims and assist them with counselling referrals [6-8]. This might then prevent rape and sexual abuse victims from developing long term psychological and psychosexual sequelae, which are very costly to treat, both in terms of finance and clinic time [2-5].

\section{References}

1. www.medway.gov.uk

2. Howard A, Rigers S, Campbell J (2003) Counselling services and battered women-a comparison of outcomes for physical and sexual assault survivors. J Interpers Violence 18: 717-734

3. Resick PA, Jordan CG, Girelli SA, Hutter CK, Marhoefer-Dvorak S (1988) A comparative outcome study of behaviour group therapy for sexual assault victims. Behavior Therapy 19: 385-401.

4. Skeketee G (1987) Rape victims, post-traumatic stress responses and their treatment; a review of the literature. J Anxiety Disorders1: 69-86.

5. www.zoelodrick.co.uk

6. www.cps.gov.uk

7. Evaluation of ISVAs and IDVAs.

8. Robinson A, Home Office (2009) Independent-Sexual-Violence-Advisors. A process evaluation. 